\title{
Acute oesophageal symptoms
}

\author{
Authors: SM Noor Hossain ${ }^{A}$ and John de Caestecker ${ }^{B}$
}

Acute oesophageal symptoms include acute dysphagia or food bolus impaction (most commonly due to strictures, Schatzki ring and eosinophilic oesophagitis), acute chest pain with odynophagia due to oesophageal infections, motility disorders and acute oesophageal rupture (of which oesophageal intramural haematoma is a subtype). Acute full thickness oesophageal rupture carries a high mortality if not recognised early; the clinical features and conditions with which this may be confused are presented and discussed.

\section{Acute oesophageal symptoms: definitions and causes}

This review covers presentation and management of acute dysphagia, odynophagia and oesophageal chest pain.

Dysphagia means 'difficulty in swallowing', distinguished from odynophagia ('pain on swallowing') by a sensation of bolus arrest/ delay, localised anywhere between cricoid and xiphisternum. The sensation of obstruction is associated with swallowing, differentiated from globus - a 'blockage' or 'lump' between cricoid and sternal notch independent of swallowing, typically persisting for hours ${ }^{1}$ - and early satiety. The latter is often described as food 'lying' in the epigastrium or the retrosternal area but differing from dysphagia, which arises during swallowing, whereas the fullness of early satiety builds during a meal, eventually inhibiting intake. Food bolus obstruction is sudden-onset total dysphagia, discussed in more detail below.

Oesophageal pain resembles other intra-thoracic/upper abdominal visceral pain (eg heart, aorta, gallbladder or lungs), not surprisingly since sensory pathways from these organs and chest wall enter the thoracic spinal cord at similar levels. Thus, oesophageal pain may occur in the chest, epigastrium, back, jaws or $\operatorname{arm}(\mathrm{s})$, and is more likely when dysphagia or odynophagia are present.

Common causes of acute oesophageal symptoms are listed in Box 1.

\section{Food bolus impaction}

Food bolus impaction is commonest in younger adults. In total, $88-97 \%$ of all patients with food bolus impaction have underlying oesophageal abnormalities. ${ }^{2}$ Schatzki ring

Authors: Aspecialist registrar in gastroenterology, University Hospitals of Leicester, Leicester, UK; B Consultant gastroenterologist, University Hospitals of Leicester, Leicester, UK

\section{Box 1. Causes of acute oesophageal symptoms.}

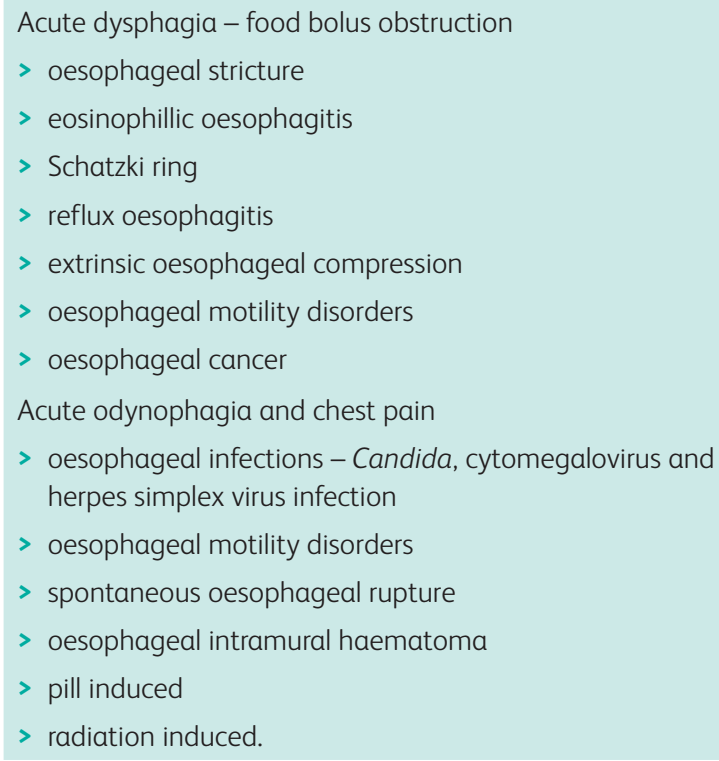

and peptic stricture are the commonest abnormalities ${ }^{2-4}$ (Table 1), followed by eosinophilic oesophagitis (EOE), which accounts for over $50 \%$ of food impactions in some published

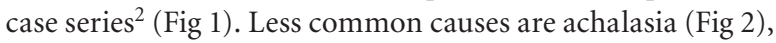
reflux oesophagitis, extrinsic oesophageal compression and oesophageal cancer.

Food bolus impactions are acute events immediately recognised by sufferers. Impactions at cricoid level may be

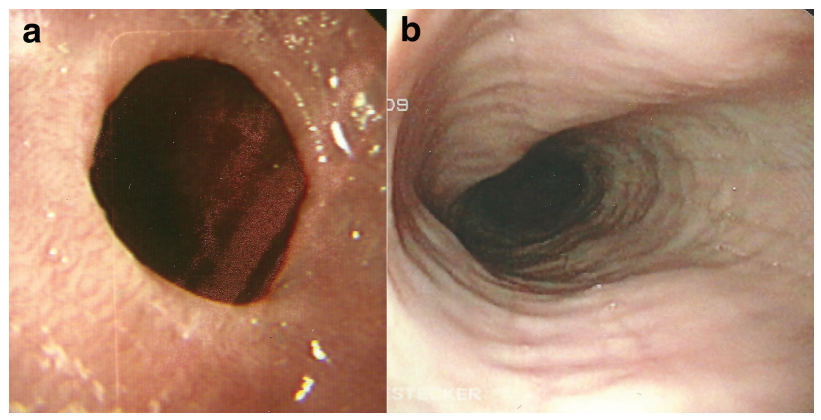

Fig 1. Endoscopic appearances of (a) Schatzki ring, a ring at the gastro-oesophageal mucosal junction above a small sliding hiatus hernia, and (b) eosinophilic oesophagitis with rings and subtle longitudinal oesophageal furrows. 


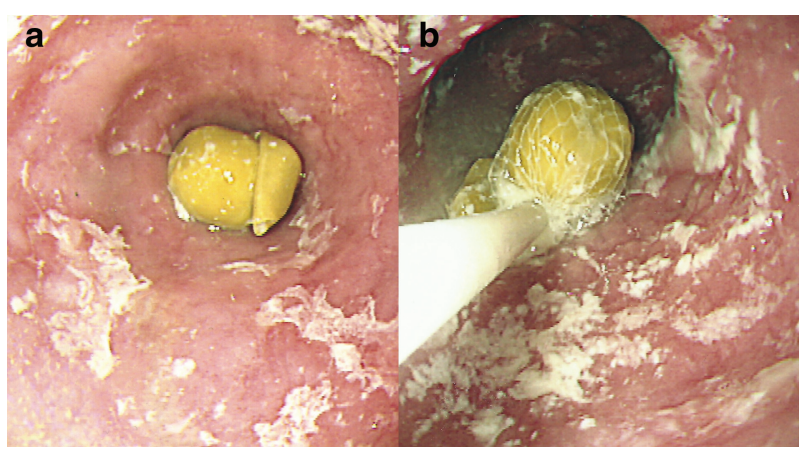

Fig 2. (a) Food bolus obstruction lasting 36 hours due to a pea in an elderly patient with known achalasia and (b) removal of the pea with a Roth net. Following removal the patient was treated with $80 \mathrm{U}$ botulinum toxin into the tight lower oesophageal sphincter.

accompanied by choking, whereas impaction in the lower oesophagus may be perceived in the upper chest or base of neck. Typically during a meal containing meat, patients report a sudden inability to swallow solids or liquids (even saliva). The impacted bolus is regurgitated or passes on spontaneously after minutes or hours, but $10-20 \%$ remain impacted. ${ }^{2}$ Associated features include choking, excessive salivation, neck or retrosternal pain, vomiting and retching. Episodes may recur infrequently -for years in patients with EOE or Schatzki ring but recurrent (non-impaction) dysphagia raises the possibility of underlying benign or malignant stricture, the latter typically in older patients with a shorter history of progressive dysphagia.

\section{Key points}

Differentiate dysphagia from globus and early satiety.

Food bolus obstruction occurs commonly with oesophageal strictures, Schatzki rings and eosinophilic oesophagitis.

Eosinophilic oesophagitis may present with normal endoscopy and requires biopsies from upper and lower oesophagus for diagnosis.

Proton pump inhibitors can effectively treat many cases of both Schatzki ring and eosinophilic oesophagitis without the need for endoscopic dilatation.

Oesophageal motility disorders rarely present with food bolus obstruction but a prior history of intermittent dysphagia for liquids and solids and normal endoscopy is frequent.

Acute oesophageal rupture needs to be diagnosed early to reduce mortality and can mimic several acute chest conditions.

KEYWORDS: Food bolus obstruction, oesophageal rupture, oesophageal infections, oesophageal motility disorders
Intermittent dysphagia for liquids and solids suggests a motility disorder. A history of atopy or allergy may accompany EOE.

Patients with recurrent food bolus obstruction learn corrective measures, eg carbonated drinks, or manoeuvring the neck or torso into particular positions. During unresolved impaction, airway patency and risk of aspiration should be assessed. Fever, tachycardia, and/or surgical emphysema in the neck suggests perforation.

\section{Management}

Plain radiography rarely helps as most food is radiolucent, but chest X-ray may suggest perforation (pneumomediastinum); contrast radiography risks aspiration. Patients without complications should undergo endoscopy, the timing of which depends on clinical features. Excessive salivation and aspiration risk require emergency endoscopy; otherwise endoscopy should be performed within 6-12 hours. Glucagon $1.0 \mathrm{mg}$ intravenously (to relax oesophageal smooth muscle) has been advocated to allow spontaneous passage of impacted food. Though studies show success rates of $12-50 \%$, a randomised trial failed to substantiate this. ${ }^{2}$ The food bolus can be extracted endoscopically using a Roth net, en bloc or piecemeal (Fig 2), or gently pushed into the stomach, sometimes after breaking it up with a wire snare. In two studies of 375 patients with impaction, the push technique was successful in $84-97 \%$ with no perforations. ${ }^{3-5}$ Endoscopy may detect Schatzki ring or peptic stricture, and allow endoscopic dilation, though in both (unless the stricture is severe) improvement often occurs with proton pump inhibitors (PPIs).

Endoscopy may show features of EOE (oesophageal rings, linear furrows (Fig 1) and mucosal fragility), but may be normal in 20-30\%; two biopsies should be taken from the distal and upper oesophagus even with normal endoscopy. A histopathological finding of $\geq 15$ eosinophils per high power field is diagnostic. ${ }^{6}$ In total, $30-40 \%$ of EOE patients respond to PPIs; ${ }^{7}$ the remainder require repeated courses of swallowed topical steroids (eg budesonide or fluticasone) or systemic steroids. ${ }^{8}$ Hypoallergenic diets (elemental and elimination) are also effective. ${ }^{9}$

\section{Oesophageal dysmotility}

In the context of acute chest pain and dysphagia, oesophageal motility disorders should be considered when endoscopy and oesophageal biopsies are normal. Food bolus obstruction occurs rarely with achalasia (Fig 2) or oesophageal spasm, usually on a background of intermittent dysphagia and regurgitation for liquids and solids. ${ }^{1,2}$ Achalasia symptoms may be severe enough to cause weight loss, whereas oesophageal spasm presents with intermittent chest pain (often resembling angina) and dysphagia. While barium-swallow radiology and endoscopy may show characteristic appearances for achalasia (dilated oesophagus with food residue, tight lower oesophageal sphincter), these may be normal.

Modern diagnosis relies on high-resolution manometry with specific metrics and an algorithm (Chicago classification $)^{10}$ categorising motility disorders into disorders of oesophagogastric outflow obstruction (including three achalasia variants), major disorders of peristalsis (including oesophageal spasm) and minor peristaltic disorders (see Fig 3). 
a

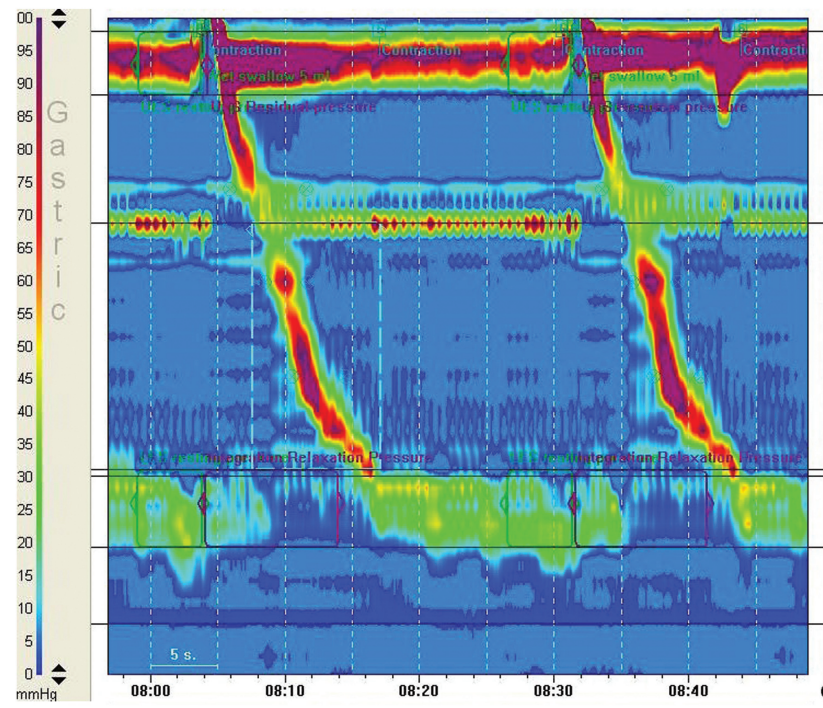

b

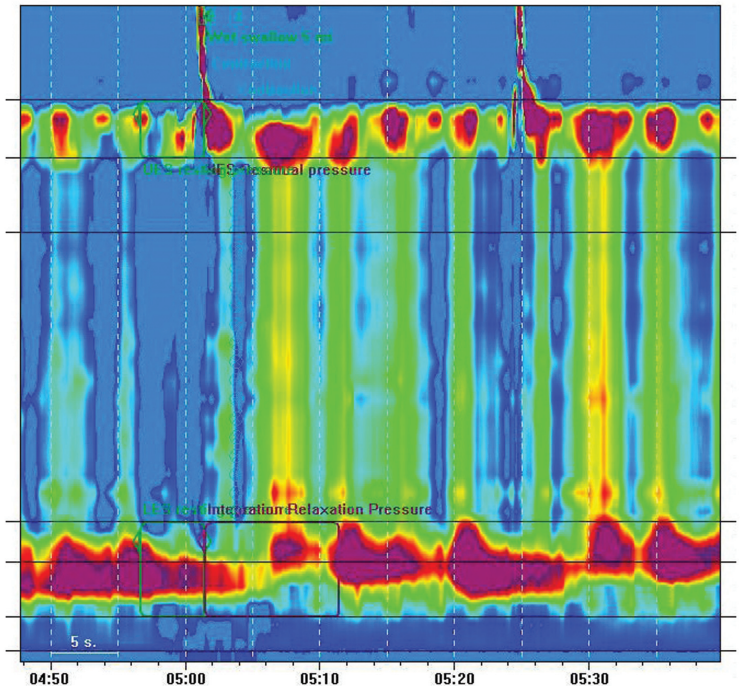

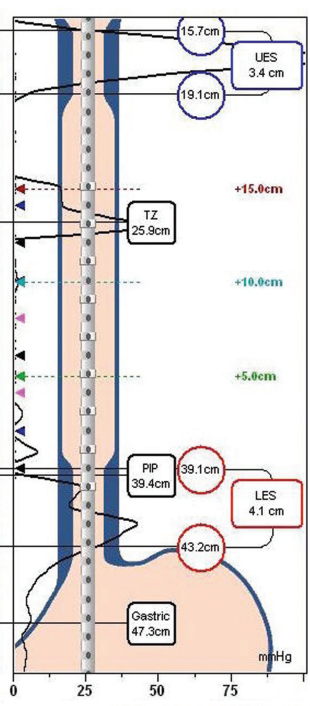

C

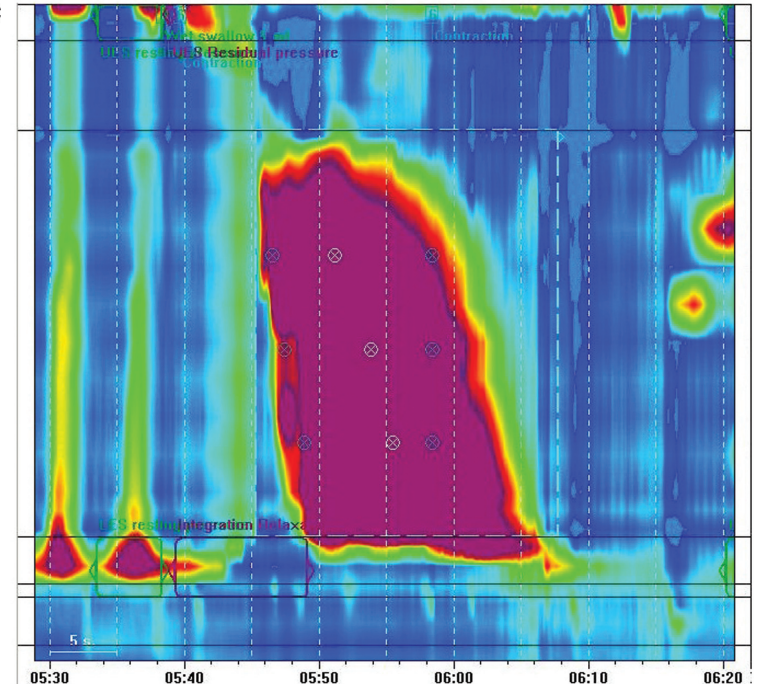

Fig 3. High-resolution oesophageal manometry with topographical maps of oesophageal motility. X axis shows time (10-second intervals), Y axis shows oesophageal length with UES as a continuous bar above and LES below. Both sphincters relax with swallowing. The colours indicate pressure (bluegreen lowest, red-purple highest. (a) Normal; (b) achalasia with non-peristaltic pan-oesophageal pressurisation and failed/incomplete LES relaxation; and (c) diffuse spasm - some non-peristaltic waves, a high pressure prolonged duration wave, normal LES relaxation. LES = lower oesophageal sphincter; TZ= transitional zone (aortic impression); UES = upper oesophageal sphincter.

\section{Management of achalasia and oesophageal spasm}

Treatment of achalasia is by lowering lower oesophageal sphincter pressure. Pharmacotherapy has a limited role; however, sublingual nifedipine may give short-term relief. Botulinum toxin injection into the lower oesophageal sphincter is effective in $85 \%$ cases, but effects diminish with time; only $30 \%$ remain improved at 2 years. ${ }^{11}$ Repeated botulinum injection is useful for those unfit for surgery. ${ }^{11}$ Others can be offered graded 30-40 mm pneumatic balloon dilation (2-3\% risk of oesophageal perforation) or laparoscopic Heller's myotomy. Dilation and surgery have comparable success rates, ${ }^{12}$ and therefore choice depends on patient preference and institutional expertise. Peroralendoscopic myotomy (POEM) is a new endoscopic procedure but experience is currently limited. ${ }^{13}$
For oesophageal spasm, diagnose and treat gastrooesophageal (GO) reflux (if present). If GO reflex is absent, smooth muscle relaxants may work if given with symptoms (eg glyceryltrinitrate spray or sublingual nifedipine), but regular dosing is less effective. Low-dose tricyclic agents (eg imipramine 50-100 mg at night) may reduce chest pain, though the use of agents is often limited by side effects. Other options include botulinum toxin into the distal $10 \mathrm{~cm}$ of the oesophageal wall (for predominant dysphagia symptoms), ${ }^{14}$ sildenafil ${ }^{15}$ or rarely, surgical long myotomy or POEM. ${ }^{16}$

\section{Oesophageal infection}

Infectious oesophagitis presents with acute odynophagia and chest pain, sometimes with fever and vomiting. ${ }^{17}$ The commonest agent is Candida albicans. Two other important 
organisms are cytomegalovirus (CMV) and herpes simplex virus (HSV). All may occur with impairment of normal physiology (salivation and oesophageal motility), but most commonly with immunosuppression (eg HIV, diabetes, malnutrition, cancer or immunosuppressant use). HSV can occur in young immunocompetent individuals.

Oesophageal candidiasis often presents without oral thrush, especially among patients using inhaled steroids. Endoscopy is usually needed for diagnosis. Management should be with systemic antifungals (eg fluconazole 50-100 mg daily for 1-2 weeks). ${ }^{17}$

Endoscopic appearances of circumscribed punched-out oesophageal ulcers with raised edges are characteristic of HSV oesophagitis, confirmed by biopsies showing multinucleate giant cells, with ground-glass nuclei and eosinophilic inclusions. Aciclovir is the treatment of choice. ${ }^{17} \mathrm{CMV}$ oesophagitis appears as large (often linear) deep ulcers in the mid or lower oesophagus with biopsies showing intranuclear/ intracytoplasmic inclusions. Ganciclovir is the treatment of choice. $^{17}$

\section{Spontaneous oesophageal rupture}

First described in 1724 by Herman Boerhaave, whose name is eponymous with the condition, spontaneous rupture of the oesophagus occurs with sudden increases in intraoesophageal pressure with negative intrathoracic pressure. It is part of a spectrum including Mallory-Weiss tear and oesophageal intramural haematoma. ${ }^{18} 15 \%$ of oesophageal perforations are spontaneous with a prevalence of $3.1 / 1,000,000 /$ year. ${ }^{19}$ This rare condition carries a mortality of approximately $35 \%$, but a delay in diagnosis of 12-24 hours increases mortality to over $60 \% .{ }^{19}$ Forceful vomiting is the commonest cause, followed by seizures, prolonged coughing, weight lifting, straining at defecation, food binging and laughing.

Mackler's diagnostic triad includes vomiting, chest pain and subcutaneous emphysema, but some of these classic features may be absent. Severe chest pain, often radiating to the back, is typical, though occasionally absent. Subcutaneous emphysema takes at least one hour to become apparent. Other features include odynophagia, tachycardia and tachypnoea. Early diagnosis is likeliest if the possibility is entertained; differential diagnoses include myocardial infarction, pulmonary embolism, pneumonia and aortic dissection $-30 \%$ are initially misdiagnosed. ${ }^{20}$ Chest X-ray may show pneumomediastinum and/or pleural effusion (often left sided), but may be normal; computerised tomography (CT) chest scan with oral and intravenous contrast allows precise diagnosis.

Management includes early review by an oesophagogastric surgeon to consider emergency operative repair or oesophagectomy; established intrathoracic contamination/ sepsis with delayed diagnosis requires antibiotics/antifungals, drainage of sepsis and sealing of the perforation with a covered removable oesophageal stent ${ }^{21}$ or more recently by endoscopic clips. ${ }^{22}$ This may be best for patients unfit for surgery, who require intensive care unit management and parenteral feeding.

\section{Oesophageal intramural haematoma}

Oesophageal intramural haematoma has been reported to occur most frequently among patients with coagulopathies or using anticoagulants or antiplatelet agents; it may also occur spontaneously, or as a result of a sudden increase in intraoesophageal pressure, or following endoscopic procedures. It presents with acute chest/back pain, dysphagia/odynophagia and haematemesis. Diagnosis is by CT scan showing a nonenhancing oesophageal mass, and/or endoscopy showing a smooth bluish oesophageal mucosal bulge. ${ }^{18}$ Management is supportive, including correcting clotting disturbances (if present). The oesophageal mucosa overlying the haematoma usually sloughs off within 2-3 weeks, leaving a long linear ulcer. $^{18}$

\section{Concluding remarks}

Acute oesophageal symptoms require a careful history: dysphagia may be confused with globus and with early satiety. Acute food bolus obstruction is readily recognised but the underlying causes may be subtle (Schatzki ring, eosinophilic oesophagitis or motility disorders) and easily missed at endoscopy. Acute chest pain and odynophagia may be due to motility disorders and oesophageal infections, but acute oesophageal rupture and a less severe variant (oesophageal intramural haematoma) are easily overlooked if not considered early in the differential diagnosis of acute chest pain.

\section{References}

1 Cook IJ. Diagnostic evaluation of dysphagia. Nat Clin Pract Gastroenterol Hepatol 2008;5:393-403.

2 Ko HH, Enn R. Review of food bolus management. Can J Gastroenterol 2008;22:805-8.

3 Vicari JJ, Johanson JF, Frakes JT. Outcomes of acute esophageal food impaction: success of the push technique. Gastrointest Endosc 2001;53:178-81.

4 Longstreth GF, Longstreth KJ, Yao JF. Esophageal food impaction: epidemiology and therapy. A retrospective, observational study. Gastrointest Endosc 2001;53:193-8.

5 Ikenberry SO, Jue TL, Anderson MA et al. Management of ingested foreign bodies and food impactions: ASGE Guidelines. Gastrointest Endosc 2011;73:1085-91.

6 Dellon ES, Gonsalves N, Hirano I et al. ACG clinical guideline: evidenced based approach to the diagnosis and management of esophageal eosinophilia and eosinophilic esophagitis (EoE). Am J Gastroenterol 2013;108:679-92.

7 Molina-Infante J, Katzka DA, Dellon ES. Proton pump inhibitor responsive esophageal eosinophilia: A historical perspective on a novel and evolving entity. Rev Esp Enferm Dig 2015 107:29-36.

8 Sawas T, Dhalla S, Sayyar M, Pasricha PJ, Hernaez R. Systematic review with meta-analysis: pharmacological interventions for eosinophilic oesophagitis. Aliment Pharmacol Therap 2015, epub ahead of print.

9 Arias A, Gonzalez-Cervera J, Tenias JM et al. Efficacy of dietary interventions for inducing histologic remission in patients with eosinophilic esophagitis: a systematic review and meta-analysis. Gastroenterology 2014;146:1639-48.

10 Kahrilas PJ, Bredenoord AJ, Fox M et al. The Chicago classification of esophageal motility disorders, v3.0. Neurogastroenterol Motil 2015;27:160-74.

11 Martínek J, Siroký M, Plottová Z et al. Treatment of patients with achalasia with botulinum toxin: a multicenter prospective cohort study. Dis Esophagus 2003;16:204-9.

12 Boeckxstaens GE, Annese V, des Varannes SB et al. Pneumatic dilation versus laparoscopic Heller's myotomy for idiopathic achalasia. N Engl J Med 2011;364:1807-16 . 
13 Talukdar R, Inoue H, Reddy DN. Efficacy of peroral endoscopic myotomy (POEM) in the treatment of achalasia: a systematic review and meta-analysis. Surg Endosc 2014, epub ahead of print.

14 Vanuytsel T, Bisschops R, Farré R et al. Botulinum toxin reduces Dysphagia in patients with non-achalasia primary esophageal motility disorders. Clin Gastroenterol Hepatol 2013;11:1115-21.

15 Fox M, Sweis R, Wong T, Anggiansah A. Sildenafil relieves symptoms and normalizes motility in patients with oesophageal spasm: a report of two cases. Neurogastroenterol Motil 2007;19:798-803.

16 Louis H, Covas A, Coppens E, Devière J. Distal esophageal spasm treated by peroral endoscopic myotomy. Am J Gastroenterol 2012;107:1926-7.

17 Rosołowski M, Kierzkiewicz M. Etiology, diagnosis and treatment of infectious esophagitis. Prz Gastroenterol 2013;8:333-7.

18 Jeong ES, Kim MJ, Yoo SH et al. Intramural hematoma of the esophagus after endoscopic pinch biopsy. Clin Endosc 2012;45:417-20.
19 Blencowe NS, Strong S, Hollowood AD. Spontaneous oesophageal rupture. BMJ 2013;346:f3095.

20 Griffin SM, Lamb PJ, Shenfine J et al. Spontaneous rupture of the oesophagus. Br J Surg 2008;95:1115-20.

21 Schweigert M, Beattie R, Solymosi N et al. Endoscopic stent insertion versus primary operative management for spontaneous rupture of the esophagus (Boerhaave syndrome): an international study comparing the outcome. Am Surg 2013;79:634-40.

22 Kobara H, Mori H, Rafiq K et al. Successful endoscopic treatment of Boerhaave syndrome using an over-the-scope clip. Endoscopy 2014;46:E82-3.

Address for correspondence: $\mathrm{Dr}]$ de Caestecker, Leicester General Hospital, Gwendolen Road, Leicester LE5 4PW, UK. Email: john.decaestecker@uhl-tr.nhs.uk

\title{
Acute upper gastrointestinal bleeding
}

\author{
Authors: Matthew Kurien ${ }^{A}$ and Alan J Lobo ${ }^{B}$
}

Acute upper gastrointestinal bleeding (AUGIB) is a frequently encountered medical emergency with an incidence of 84$160 / 100,000$ and associated with mortality of approximately $10 \%$. Guidelines from the National Institute for Care and Care Excellence outline key features in the management of AUGIB. Patients require prompt resuscitation and risk assessment using validated tools. Upper gastrointestinal endoscopy provides accurate diagnosis, aids in estimating prognosis and allows therapeutic intervention. Endoscopy should be undertaken immediately after resuscitation in unstable patients and within 24 hours in all other patients. Interventional radiology may be required for bleeding unresponsive to endoscopic intervention. Drug therapy depends on the cause of bleeding. Intravenous proton pump inhibitors should be used in patients with highrisk ulcers. Terlipressin and broad-spectrum antibiotics should be used following variceal haemorrhage. Hospitals admitting patients with AUGIB need to provide well organised services and ensure access to relevant services for all patients, and particularly to out of hours endoscopy.

Authors: Aacademic clinical lecturer in gastroenterology, Department of Gastroenterology, Royal Hallamshire Hospital, Sheffield, UK, and Academic Unit of Gastroenterology, University of Sheffield, Beech Hill Rd, Sheffield, UK; ${ }^{B}$ consultant gastroenterologist, Sheffield Teaching Hospitals NHS Foundation Trust, Sheffield, UK, and professor of gastroenterology, Academic Unit of Gastroenterology, University of Sheffield, Sheffield, UK

\section{Introduction}

Acute upper gastrointestinal bleeding (UGIB) is a common medical emergency characterised by haematemesis and/or melaena. Massive haemorrhage from the upper GI tract may be associated with brighter rectal bleeding. Haemodynamic instability may also feature, with patients presenting with dizziness, syncope or in hypovolaemic shock. Despite the declining incidence of UGIB, advances in therapeutic endoscopy and increased use of acid suppressing medication, there is a significant in-hospital mortality. ${ }^{1}$ This review provides an overview of UGIB incorporating current standards for its management (Box 1).

\section{Epidemiology}

The incidence of UGIB is between 84 and 160 cases per 100,000 adults in European populations, with incidence highest in men, in lower socioeconomic groups and in the elderly. ${ }^{2}$ Peptic ulcer bleeding is the commonest identified cause, though its incidence is declining - possibly due to the use of proton pump inhibitors (PPIs) and the decreasing prevalence of Helicobacter pylori infection. By contrast, the relative contribution of variceal bleeding appears to be rising in the UK, accounting for $11 \%$ of all UGIB admissions in the 2007 national audit, ${ }^{1}$ compared to only $4 \%$ in $1993 .{ }^{3}$ Mortality in the successive national audits has appeared to fall from $14 \%$ to $10 \% .{ }^{1,3}$

\section{Pre-endoscopy care}

\section{Assessment and risk stratification}

All patients presenting with UGIB require prompt assessment using a validated assessment tool. Early assessment identifies 